PROCEEDINGS OF THE

AMERICAN MATHEMATICAL SOCIETY

Volume 137, Number 4, April 2009, Pages 1295-1302

S 0002-9939(08)09638-X

Article electronically published on October 29, 2008

\title{
POWERS OF COXETER ELEMENTS IN INFINITE GROUPS ARE REDUCED
}

\author{
DAVID E. SPEYER
}

(Communicated by Jim Haglund)

\begin{abstract}
Let $W$ be an infinite irreducible Coxeter group with $\left(s_{1}, \ldots, s_{n}\right)$ the simple generators. We give a short proof that the word $s_{1} s_{2} \cdots s_{n} s_{1} s_{2} \cdots$ $s_{n} \cdots s_{1} s_{2} \cdots s_{n}$ is reduced for any number of repetitions of $s_{1} s_{2} \cdots s_{n}$. This result was proved for simply laced, crystallographic groups by Kleiner and Pelley using methods from the theory of quiver representations. Our proof uses only basic facts about Coxeter groups and the geometry of root systems. We also prove that, in finite Coxeter groups, there is a reduced word for $w_{0}$ which is obtained from the semi-infinite word $s_{1} s_{2} \cdots s_{n} s_{1} s_{2} \cdots s_{n} \cdots$ by interchanging commuting elements and taking a prefix.
\end{abstract}

Let $W$ be a Coxeter group with $S$ the generating set of simple reflections. An element $c \in W$ of the form $s_{1} \cdots s_{n}$, with $s_{1}, \ldots, s_{n}$ some ordering of the elements of $S$, is called a Coxeter element. It is a result of Howlett 4 that, if $W$ is infinite, then any Coxeter element has infinite order. Our main result is a dramatic strengthening of this result:

Theorem 1. Let $W$ be an infinite, irreducible Coxeter group and let $\left(s_{1}, \ldots, s_{n}\right)$ be any ordering of the simple generators. Then the word $s_{1} \cdots s_{n} s_{1} \cdots s_{n} \cdots s_{1} \cdots s_{n}$ is reduced for any number of repetitions of $s_{1} \cdots s_{n}$.

There are several earlier results which are special cases of Theorem 1] In 9, it is shown that, in each classical affine group, there is an ordering $\left(s_{1}, \ldots, s_{n}\right)$ of the simple generators such that the word $s_{1} \cdots s_{n} s_{1} \cdots s_{n} \cdots s_{1} \cdots s_{n}$ is reduced for any number of repetitions of $s_{1} \cdots s_{n} 1$ Fomin and Zelevinsky [2, Corollary 9.6] proved a version of Theorem 1 for Coxeter groups with bipartite diagrams; they show that, if $S=I \sqcup J$ is a partition of $S$ into two sets so that all the elements in each set commute, and if $W$ is irreducible and infinite, then the word $\prod_{i \in I} s_{i} \prod_{j \in J} s_{j} \prod_{i \in I} s_{i} \prod_{j \in J} s_{j} \cdots \prod_{i \in I} s_{i} \prod_{j \in J} s_{j}$ is reduced for any number of repetitions of $\prod_{i \in I} s_{i} \prod_{j \in J} s_{j}$. Krammer [8] defines an element $w$ of $W$ to be straight if $\ell\left(w^{m}\right)=|m| \ell(w)$ for all integers $m$. In his terminology, $w$ is straight if and only if the identity element is contained in the axis of $w$. This gives a finite procedure to check whether any particular $w$ is straight, but it is not clear how

Received by the editors February 11, 2008, and, in revised form, May 8, 2008.

2000 Mathematics Subject Classification. Primary 20F55.

${ }^{1}$ More specifically, the authors of [9] define four properties of a sequence $r_{1}, r_{2}, \ldots$ of simple reflections; property (IV) is that the word $r_{1} r_{2} \cdots r_{N}$ is reduced for any $N$. For each classical affine type, they exhibit a sequence of reflections which satisfies their properties and the ordering is periodic in each case.

(C)2008 American Mathematical Society Reverts to public domain 28 years from publication 
Krammer's methods could be used to show that infinitely many elements, in different Coxeter groups, are straight. It is trivial to extend any of these results to the case where $W \cong W_{1} \times W_{2} \times \cdots \times W_{r}$, with each $W_{i}$ a Coxeter group meeting the above conditions.

Most relevant to us is recent work of Kleiner and Pelley [5], who show that if $W$ is a simply laced, crystallographic Coxeter group which is irreducible and infinite, then the word $s_{1} \cdots s_{n} s_{1} \cdots s_{n} \cdots s_{1} \cdots s_{n}$ is reduced for any number of repetitions of $s_{1} \cdots s_{n}$. Their argument relies heavily on the theory of quiver representations, and on the work of Kleiner and Tyler [6]. Our proof is inspired by that of Kleiner and Pelley, but we strip out the quiver theory and simplify several arguments. We also eliminate the need for the assumptions that $W$ is crystallographic and simply laced.

The essential property of infinite Coxeter groups, in this proof, is that they have no maximal element. Therefore, it is reasonable to guess that our methods can be used to prove some property of the maximal element, $w_{0}$, in a finite Coxeter group. One guess might be that we could prove that there is some prefix of the semiinfinite word $s_{1} s_{2} \cdots s_{n} s_{1} s_{2} \cdots s_{n} \cdots$ which forms a reduced word for $w_{0}$, so that the partial products of this word climb all the way to the top of $W$ before becoming nonreduced. This isn't quite true, but the following variant is: It is possible to interchange commuting elements of $s_{1} s_{2} \cdots s_{n} s_{1} s_{2} \cdots s_{n} \cdots$ so that a reduced word for $w_{0}$ appears as a prefix. For example, let $W$ be the symmetric group on four elements and let $s_{1}=(12), s_{2}=(23)$ and $s_{3}=(34)$. Then we can swap the second occurrence of $s_{3}$ with the third occurrence of $s_{1}$ in $s_{1} s_{2} s_{3} s_{1} s_{2} s_{3} s_{1} s_{2} s_{3} \cdots$, and the reduced word $s_{1} s_{2} s_{3} s_{1} s_{2} s_{1}$ for $w_{0}$ will appear as a prefix. We prove that this is always possible in Corollary 4.1 .

Our primary technical tool is the introduction of a skew-symmetric form $\omega_{c}$ on the root space. In [13], Nathan Reading and the author use this form to generalize Reading's results on sortable elements to infinite Coxeter groups.

\section{Conventions Regarding Coxeter groups}

Let $W$ be a Coxeter group of rank $n$. That means that $W$ is generated by $s_{1}, \ldots, s_{n}$, subject to the relations $s_{i}^{2}=1$ and $\left(s_{i} s_{j}\right)^{m_{i j}}=1$ for $i \neq j$, where $2 \leq m_{i j}=m_{j i} \leq \infty$. Note that the $m_{i j}$ may be any integers; we do not assume that $W$ is crystallographic. The Coxeter diagram of $W$ is the graph $\Gamma$ whose vertices are labeled $1, \ldots, n$ and where there is an edge between $i$ and $j$ if $m_{i j} \neq 2$. The group $W$ is called irreducible if $\Gamma$ is connected. An element of $W$ which is conjugate to one of the elements of $S$ is called a reflection; the elements of $S$ are called simple reflections. An element of the form $s_{x_{1}} \cdots s_{x_{n}}$ of $W$, for some permutation $x_{1} \cdots x_{n}$ of $\{1, \ldots, n\}$, is called a Coxeter element. Given such a permutation, direct $\Gamma$ such that $x_{j} \rightarrow x_{i}$ if $i<j$. Two permutations yield the same Coxeter element if and only if they give rise to the same orientation of $\Gamma$, so in this way we may identify Coxeter elements and acyclic orientations of $\Gamma 2$

Let $V$ be the $n$-dimensional real vector space with basis $\alpha_{1}, \ldots, \alpha_{n}$ and equip $V$ with the symmetric bilinear form $B$ such that $B\left(\alpha_{i}, \alpha_{i}\right)=2$ and $B\left(\alpha_{i}, \alpha_{j}\right)=$ $-2 \cos \left(\pi / m_{i j}\right)$ for $i \neq j$. Then $W$ acts on $V$ by $s_{i}: v \mapsto v-B\left(v, \alpha_{i}\right) \alpha_{i}$, and this action preserves the bilinear form $B$. The elements of $V$ of the form $w \alpha_{i}$ are called

\footnotetext{
${ }^{2}$ This identification between Coxeter elements and acyclic orientations of $\Gamma$ is the one used in [5]; it is the opposite of the one used in [10] and [11].
} 
roots 3 Every root is either in the positive real span of the $\alpha_{i}$, in which case it is called a positive root, or in the positive real span of the $-\alpha_{i}$, in which case it is called a negative root. The positive roots are in bijection with the reflections, via $w \alpha_{s} \leftrightarrow w s w^{-1}$. We write $\alpha_{t}$ for the positive root associated to the reflection $t$. We have $w \alpha_{t}= \pm \alpha_{w t w^{-1}}$.

For any $w \in W$, the set of inversions of $w$ is defined to be the set of reflections $t$ such that $w^{-1} \alpha_{t}$ is a negative root. If we write $w$ as $s_{x_{1}} \cdots s_{x_{N}}$, then the inversions of $w$ are the reflections that occur an odd number of times in the sequence $s_{x_{1}}$, $s_{x_{1}} s_{x_{2}} s_{x_{1}}, s_{x_{1}} s_{x_{2}} s_{x_{3}} s_{x_{2}} s_{x_{1}}, \ldots, s_{x_{1}} s_{x_{2}} \cdots s_{x_{n}} \cdots s_{x_{2}} s_{x_{1}}$. The length of $w$, written $\ell(w)$, is the length of the shortest expression for $w$ as a product of the simple generators and a product which achieves this minimal length is called reduced. If $s_{x_{1}} \cdots s_{x_{N}}$ is reduced, then $s_{x_{1}} \cdots s_{x_{i-1}} \alpha_{x_{i}}=\alpha_{s_{x_{1}} \cdots s_{x_{i-1}} s_{x_{i}} s_{x_{i-1}} \cdots s_{x_{1}}}$ (as opposed to $\left.-\alpha_{s_{x_{1}} \cdots s_{x_{i-1}}} s_{x_{i}} s_{x_{i-1}} \cdots s_{x_{1}}\right)$. Also, if $s_{x_{2}} \cdots s_{x_{N}}$ is reduced, then $s_{x_{1}} s_{x_{2}} \cdots s_{x_{N}}$ is reduced if and only if $s_{x_{1}}$ is not an inversion of $s_{x_{2}} \cdots s_{x_{N}}$.

The previous three paragraphs are very well known; a good reference for this material and far more concerning Coxeter groups is 1, particularly Chapters 1 and 4 . We now describe one additional combinatorial tool and one geometric tool. For $i$ between 1 and $n$, define the map $\pi_{i}: W \rightarrow W$ by $\pi_{i}(w)=s_{i} w$ if $\ell\left(s_{i} w\right)>\ell(w)$ and $\pi_{i}(w)=w$ otherwise. This is sometimes known as the degenerate Hecke action. The condition that $\ell\left(s_{i} w\right)>\ell(w)$ is equivalent to the condition that $s_{i}$ is not an inversion of $w$. If $s_{x_{1}} \cdots s_{x_{N}}$ is reduced, then $\pi_{x_{1}} \cdots \pi_{x_{N}} e=s_{x_{1}} \cdots s_{x_{N}}$. Also, if $s_{i}$ and $s_{j}$ commute, so do $\pi_{i}$ and $\pi_{j}$. We call $\pi_{x_{1}} \cdots \pi_{x_{N}} e$ the Demazure product of $x_{1} \cdots x_{n}$. For a quick introduction to the properties of the Demazure product, see Section 3 of [7.

Let $c=s_{x_{1}} \ldots s_{x_{n}}$ be a Coxeter element of $W$. A simple reflection $s$ is called initial in $c$ if it is the first letter of some reduced word for $c$ and is called final $i n c$ if it is the last letter of some reduced word for $c$. So $s_{x_{1}}$ is initial in $c$ and $s_{x_{n}}$ is final in $c$. We define a skew symmetric bilinear form $\omega_{c}$ on $V$ by $\omega_{c}\left(\alpha_{x_{i}}, \alpha_{x_{j}}\right)=$ $B\left(\alpha_{x_{i}}, \alpha_{x_{j}}\right)$ for $i<j$. (By skew-symmetry, $\omega_{c}\left(\alpha_{x_{i}}, \alpha_{x_{j}}\right)=-B\left(\alpha_{x_{i}}, \alpha_{x_{j}}\right)$ for $i>j$ and $\omega_{c}\left(\alpha_{i}, \alpha_{i}\right)=0$.) It is easy to check that $\omega_{c}$ does not depend on the choice of a reduced word for $c$.

Proposition 1.1. With the above notation:

(1) For all $v$ and $w \in V$, we have $\omega_{s_{x_{1}} c s_{x_{1}}}\left(s_{x_{1}} v, s_{x_{1}} w\right)=\omega_{c}(v, w)$.

(2) For all positive roots $\alpha_{t}, \omega_{c}\left(\alpha_{s_{x_{1}}}, \alpha_{t}\right) \leq 0$, with equality only if $s_{x_{1}}$ and $t$ commute.

(3) For all positive roots $\alpha_{t}, \omega_{c}\left(\alpha_{t}, \alpha_{s_{x_{n}}}\right) \leq 0$, with equality only if $s_{x_{n}}$ and $t$ commute.

Proof. We first check property (1). Let $c=s_{1} \cdots s_{n}$ with $s=s_{1}$. We recall the formula $s v=v-B\left(\alpha_{s}, v\right) \alpha_{s}$. It is enough to check property (1) in the case that $v$ and $w$ are simple roots, say $v=\alpha_{s_{i}}$ and $w=\alpha_{s_{j}}$ with $i<j$. We consider two cases.

\footnotetext{
${ }^{3}$ Those interested in Kac-Moody algebras and quiver theory would call these vectors real roots; those from a Coxeter-theoretic background would simply call them roots. We follow the latter convention.
} 
Case $1(i=1)$. Then

$$
\begin{aligned}
& \omega_{s c s}\left(s \alpha_{s}, s \alpha_{s_{j}}\right)=\omega_{s c s}\left(-\alpha_{s}, \alpha_{s_{j}}-B\left(\alpha_{s}, \alpha_{s_{j}}\right) \alpha_{s}\right) \\
& \quad=-\omega_{s c s}\left(\alpha_{s}, \alpha_{s_{j}}\right)=B\left(\alpha_{s}, \alpha_{s_{j}}\right)=\omega_{c}\left(\alpha_{s}, \alpha_{s_{j}}\right) .
\end{aligned}
$$

We used that $s$ is final in scs and initial in $c$ to deduce the signs in the last two equalities.

Case $2(i>1)$. Then

$$
\begin{aligned}
& \omega_{s c s}\left(s \alpha_{s_{i}}, s \alpha_{s_{j}}\right)=\omega_{s c s}\left(\alpha_{s_{i}}-B\left(\alpha_{s}, \alpha_{s_{i}}\right) \alpha_{s}, \alpha_{s_{j}}-B\left(\alpha_{s}, \alpha_{s_{j}}\right) \alpha_{s}\right) \\
& \quad=\omega_{s c s}\left(\alpha_{s_{i}}, \alpha_{s_{j}}\right)-B\left(\alpha_{s}, \alpha_{s_{i}}\right) \omega_{s c s}\left(\alpha_{s}, \alpha_{s_{j}}\right)-B\left(\alpha_{s}, \alpha_{s_{j}}\right) \omega_{s c s}\left(\alpha_{s_{i}}, \alpha_{s}\right) .
\end{aligned}
$$

Now, $s$ is final in $s c s$, so $\omega_{s c s}\left(\alpha_{s}, \alpha_{s_{j}}\right)=-B\left(\alpha_{s}, \alpha_{s_{j}}\right)$ and $\omega_{s c s}\left(\alpha_{s_{i}}, \alpha_{s}\right)=B\left(\alpha_{s_{i}}, \alpha_{s}\right)$. Thus,

$$
\begin{aligned}
-B\left(\alpha_{s}, \alpha_{s_{i}}\right) \omega_{s c s}\left(\alpha_{s}, \alpha_{s_{j}}\right)- & B\left(\alpha_{s}, \alpha_{s_{j}}\right) \omega_{s c s}\left(\alpha_{s_{i}}, \alpha_{s}\right) \\
& =B\left(\alpha_{s}, \alpha_{s_{i}}\right) B\left(\alpha_{s}, \alpha_{s_{j}}\right)-B\left(\alpha_{s}, \alpha_{s_{j}}\right) B\left(\alpha_{s_{i}}, \alpha_{s}\right)=0
\end{aligned}
$$

and we deduce that

$$
\omega_{s c s}\left(s \alpha_{s_{i}}, s \alpha_{s_{j}}\right)=\omega_{s c s}\left(\alpha_{s_{i}}, \alpha_{s_{j}}\right)=B\left(\alpha_{s_{i}}, \alpha_{s_{j}}\right)=\omega_{c}\left(\alpha_{s_{i}}, \alpha_{s_{j}}\right) .
$$

We have used that $s_{i}$ comes before $s_{j}$ in a reduced word for scs, as well as in a reduced word for $c$, to deduce the signs of the last two equalities. This concludes the proof of property (11).

We now prove property (2). Let $\alpha_{t}=\sum_{r \in S} a_{r} \alpha_{r}$. Since $\alpha_{t}$ is a positive root, all of the coefficients $a_{r}$ are nonnegative. Then, as $s$ is initial in $c$, we have $\omega_{c}\left(\alpha_{s}, \alpha_{t}\right)=\sum_{r \in S \backslash\{s\}} a_{r} B\left(\alpha_{s}, \alpha_{r}\right)$. Every term in this sum is nonpositive, so $\omega_{c}\left(\alpha_{s}, \alpha_{t}\right)$ is nonpositive, which is the first half of the claim. Now, suppose that $\omega_{c}\left(\alpha_{s}, \alpha_{t}\right)$ is zero. Then $a_{r}$ is zero whenever $r$ and $s$ don't commute. Let $J$ be the set of simple reflections which commute with $s$. So the root $\alpha_{t}$ is in the span of the set $\left\{\alpha_{r}\right\}_{r \in J}$. This implies that the reflection $t$ is in $W_{J}$, which is the subgroup of $W$ generated by $J$. (Proof: Let $p$ be a point in $V^{*}$, the dual vector space to $V$, such that $\left\langle p, \alpha_{s}\right\rangle=0$ for $s \in J$ and $\left\langle p, \alpha_{s}\right\rangle \geq 0$ for $s \notin J$. Then the contragredient action of $t$ on $V^{*}$ fixes $p$. By [1, Lemma 4.5.1], the stabilizer of $p$ is $W_{J}$.) Since every generator of $W_{J}$ commutes with $s$, so does $t$. This completes the proof of property (2); the proof of property (3) is precisely analogous.

\section{Admissible Sequences}

This section essentially recapitulates (part of) Section 2 of [5], and we will try to repeat the terminology from [5] as much as possible. Let $c$ be a Coxeter element of $W$. A sequence $x_{1}, x_{2}, \ldots, x_{N}$ of elements of $\{1,2, \ldots, n\}$ is called c-admissible if $s_{x_{1}}$ is initial in $c, s_{x_{2}}$ is initial in $s_{x_{1}} c s_{x_{1}}, s_{x_{3}}$ is initial in $s_{x_{2}} s_{x_{1}} c s_{x_{1}} s_{x_{2}}$ and so forth. This definition can be understood in a purely graph-theoretic mannner, viewing $c$ as an orientation of $\Gamma$. The condition that $s_{x}$ is initial in $c$ means that $x$ is a sink of $(\Gamma, c)$ and changing $c$ to $s_{x} c s_{x}$ means reversing all edges incident to $x$, so that $x$ changes from a sink to a source.

We put an equivalence relation on the set of admissible sequences by setting two sequences to be equivalent if they differ only by interchanging the order of nonadjacent vertices. Write $[u]$ for the equivalence class of $u$. Let $\mathfrak{S}$ denote the set of admissible sequences modulo this equivalence relation. When it is necessary to 
emphasize the dependence on $c$, we will write $\mathfrak{S}_{c}$ and say that elements of $\mathfrak{S}_{c}$ are $c$-admissible. The following obvious observation will be of repeated importance:

Proposition 2.1. If $s$ and $t$ are two vertices of $\Gamma$, connected by an edge which is $c$-oriented from $t$ to $s$, then the occurrences of $s$ and $t$ in any c-admissible sequence must alternate, with s coming first.

We can now state a result which immediately implies Theorem 1.

Theorem 2. Let $W$ be an infinite, irreducible Coxeter group and let $c$ be a Coxeter element. Let $x_{1} x_{2} \ldots x_{N}$ be any c-admissible sequence. Then $s_{x_{1}} \cdots s_{x_{N}}$ is reduced.

The rest of the paper is devoted to the proof of Theorem 2

We need a small combinatorial lemma. For $u=\left[x_{1} \ldots x_{N}\right] \in \mathfrak{S}$, let $\phi(u)_{x}$ be the number of occurrences of $x$ in $x_{1} x_{2} \cdots x_{N}$. So $\phi(u)$ is an integer-valued function on the vertices of $\Gamma$. We put the structure of a poset on $\mathfrak{S}$ by setting $u \preccurlyeq v$ if one can choose representatives $u_{1} \ldots u_{M}$ and $v_{1} \ldots v_{N}$ for the equivalence classes $u$ and $v$ such that $M \leq N$ and $u_{i}=v_{i}$ for $i \leq M$.

Proposition 2.2. We have $u_{1} \ldots u_{M} \preccurlyeq v_{1} \ldots v_{N}$ if and only if $\phi\left(u_{1} \ldots u_{M}\right)_{x} \leq$ $\phi\left(v_{1} \ldots v_{N}\right)_{x}$ for every vertex $x$ of $\Gamma$.

This is part of [5. Proposition 3.2]; we provide a short proof.

Proof. The "only if" direction is obvious; we prove the "if" direction by induction on $M$. The base case $M=0$ is obvious. Note that $u_{1}$ is necessarily a sink of $\Gamma$. Since $\phi\left(u_{1} \ldots u_{M}\right)_{u_{1}} \leq \phi\left(v_{1} \ldots v_{N}\right)_{u_{1}}$, the vertex $u_{1}$ must occur somewhere in $v_{1} \ldots v_{N}$. Let $v_{r}$ be the first appearance of $u_{1}$. Let $w$ be any vertex neighboring $u_{1}$; we claim that $w$ does not occur among $v_{1}, v_{2}, \ldots, v_{r-1}$. This is because, as noted above, the occurrences of $u_{1}$ and $w$ in $v_{1} \ldots v_{N}$ must alternate, with $u_{1}$ appearing first. So $v_{1} \ldots v_{N}$ is equivalent to $v_{r} v_{1} v_{2} \ldots v_{r-1} v_{r+1} \ldots v_{N}$. By induction, $u_{2} u_{3} \ldots u_{M} \preccurlyeq$ $v_{1} v_{2} \ldots v_{r-1} v_{r+1} \ldots v_{N}$ in $\mathfrak{S}_{s_{u_{1}} c s_{u_{1}}}$, so $u_{1} \ldots u_{M} \preccurlyeq v_{1} \ldots v_{N}$ in $\mathfrak{S}_{c}$.

Remark. Kleiner and Pelley characterize the image of $\phi$ in $\mathbb{Z}^{n}$ and use it to show that the poset $\mathfrak{S}$ is a distributive semi-lattice. Hohlweg, Lange, and Thomas, in [3], study the lower interval of reduced words in $\mathfrak{S}$, in the case that $W$ is finite, and show that it is a distributive lattice as well. Hopefully, these lattices are related to the appearance of lattice theory in Nathan Reading's and the author's work. (See [10, [1], 12].)

\section{The CRUCial Lemmas}

Now, let $W$ be a Coxeter group and $\Gamma$ its Dynkin diagram. As discussed above, there is a bijection between Coxeter elements of $W$ and acyclic orientations of $\Gamma$, and we will feel free to use the same symbol to refer both to an orientation and the corresponding Coxeter element. In this section, we will establish the following.

Proposition 3.1. Let $x_{1} \cdots x_{N}$ be of minimal length among all c-admissible sequences with Demazure product $w$. Then the word $s_{x_{1}} \cdots s_{x_{N}}$ is reduced and $w=$ $s_{x_{1}} \cdots s_{x_{N}}$.

Note that, at this point, we have not made any assumptions about $W$ being infinite or irreducible. That will come later, when we apply this result to prove that particular words are reduced. The key technical trick of this note is contained in the following lemma, which will be essential in the proof of Proposition 3.1. 
Lemma 3.2. Suppose that $x_{1} \ldots x_{N}$ is c-admissible and $s_{x_{1}} \ldots s_{x_{N}}$ is a reduced word of $W$. Let $t_{i}$ be the reflection $s_{x_{1}} \cdots s_{x_{i-1}} s_{x_{i}} s_{x_{i-1}} \cdots s_{x_{1}}$. Then $\omega_{c}\left(\alpha_{t_{i}}, \alpha_{t_{j}}\right) \leq$ 0 for $i<j$, and equality implies that $t_{i}$ and $t_{j}$ commute.

Proof. Our proof is by induction on $i$. If $i=1$, then $x_{1}$ is a sink of $c$ and the result is part (2) of Proposition [1.1. If $i>1$, then, by induction, we have $\omega_{s_{x_{1}} c s_{x_{1}}}\left(\alpha_{s_{x_{1}} t_{i} s_{x_{1}}}, \alpha_{s_{x_{1}} t_{j} s_{x_{1}}}\right) \leq 0$, with equality implying that $s_{x_{1}} t_{i} s_{x_{1}}$ and $s_{x_{2}} t_{j} s_{x_{2}}$ commute. But, since $s_{x_{1}} \cdots s_{x_{N}}$ is reduced, we know that $\alpha_{s_{x_{1}} t_{i} s_{x_{1}}}=s_{x_{1}} \alpha_{t_{i}}$ and $\alpha_{s_{x_{1}} t_{j} s_{x_{1}}}=s_{x_{1}} \alpha_{t_{j}}$. By part (11) of Proposition 1.1, we have $\omega_{c}\left(\alpha_{t_{i}}, \alpha_{t_{j}}\right)=$ $\omega_{s_{x_{1}} c s_{x_{1}}}\left(s_{x_{1}} \alpha_{t_{i}}, s_{x_{1}} \alpha_{t_{j}}\right) \leq 0$ as desired. Moreover, $t_{i}$ and $t_{j}$ commute if and only if $s_{x_{1}} t_{i} s_{x_{1}}$ and $s_{x_{2}} t_{j} s_{x_{2}}$ do.

We now begin the proof of Proposition 3.1. Our proof is by induction on $N$; if $N=1$ the result is trivial. Let $w$ and $x_{1} \cdots x_{N}$ be as in the statement of Proposition 3.1 with $N>1$ and assume that the result is known, for all $c$, for all smaller values of $N$. Abbreviate $s=s_{x_{1}}$ and $w^{\prime}=\pi_{x_{2}} \cdots \pi_{x_{N}} e$. We note that $x_{2} \cdots x_{N}$ is of minimal length among scs-admissible sequences with Demazure product $w^{\prime}$; if $y_{2} \cdots y_{M}$ were a shorter such sequence, then $x_{1} y_{2} \cdots y_{M}$ would be a shorter $c$ admissible sequence with Demazure product $w$. So, by induction, $s_{x_{2}} \cdots s_{x_{N}}$ is reduced and is equal to $w^{\prime}$. The only way that $s_{x_{1}} s_{x_{2}} \cdots s_{x_{N}}$ might not be reduced then is if $s$ is an inversion of $w^{\prime}$ and $w=w^{\prime}$. We adopt the notation $u_{i}$ for $s_{x_{2}} \cdots s_{x_{i-1}} s_{x_{i}} s_{x_{i-1}} \cdots s_{x_{2}}$, where $2 \leq i \leq N$, so the $u_{i}$ are the inversions of $s_{x_{2}} \cdots s_{x_{N}}$. Suppose, for the sake of contradiction, that $s=u_{a}$ and, thus, $w=w^{\prime}$. In this case, $s s_{x_{2}} \cdots s_{x_{a-1}} s_{x_{a+1}} \cdots s_{x_{N}}=w$ and the word $s s_{x_{2}} \cdots s_{x_{a-1}} s_{x_{a+1}} \cdots s_{x_{N}}$ is reduced.

Consider any $b$ between $a+1$ and $N$. On the one hand, $\omega_{s_{x_{1}} c s_{x_{1}}}\left(\alpha_{u_{a}}, \alpha_{u_{b}}\right) \leq 0$ by Lemma 3.2. (Recall that $x_{2} \cdots x_{N}$ is reduced.) On the other hand, $u_{a}=s_{x_{1}}$ and $s_{x_{1}}$ is the final letter in $s_{x_{1}} c s_{x_{1}}$, so $\omega_{s_{x_{1}} c s_{x_{1}}}\left(\alpha_{u_{a}}, \alpha_{u_{b}}\right) \geq 0$ by part (3) of Proposition 1.1. We deduce that, for all $b$ with $a+1 \leq b \leq N$, we have $\omega_{s_{x_{1}} c s_{x_{1}}}\left(\alpha_{u_{a}}, \alpha_{u_{b}}\right)=0$ and, by Lemma 3.2 $u_{a} u_{b}=u_{b} u_{a}$. Thus, we deduce that $u_{a}$ commutes with $u_{b}$ for all $b$ with $a+1 \leq b \leq N$.

Write $v_{b}=s_{x_{a}} \cdots s_{x_{b-1}} s_{x_{b}} s_{x_{b-1}} \cdots s_{x_{a}}$. Then $s_{x_{a}}=v_{a}$ commutes with $v_{b}$ for all $b$ with $a+1 \leq b \leq N$. From the identity $s_{x_{b}}=v_{a} v_{a+1} \cdots v_{b} \cdots v_{a+1} v_{a}$, we conclude that $s_{x_{a}}$ commutes with $s_{x_{b}}$ for all $b$ between $a$ and $N$. But then $x_{1} x_{2} \cdots x_{a-1} x_{a+1} \cdots x_{N}$ is $c$-admissible. We saw above that $s s_{x_{2}} \cdots s_{x_{a-1}} s_{x_{a+1}} \cdots$ $s_{x_{N}}=w$ and the word $s s_{x_{2}} \cdots s_{x_{a-1}} s_{x_{a+1}} \cdots s_{x_{N}}$ is reduced, so $x_{1} x_{2} \cdots x_{a-1} x_{a+1} \cdots$ $x_{N}$ has Demazure product $w$. This contradicts our choice of $x_{1} x_{2} \cdots x_{a-1} x_{a} x_{a+1} \cdots$ $x_{N}$ as the shortest $c$-admissible sequence with Demazure product $w$. This contradiction concludes the proof of Proposition 3.1 .

\section{FINISHING THE PROOF}

Assume that $W$ is infinite and irreducible; recall that the second assumption simply means that the Coxeter diagram $\Gamma$ is connected. We now have a powerful tool (Proposition 3.1) to prove that certain words in $W$ are reduced. In this section, we will apply this tool to prove that $s_{x_{1}} \cdots s_{x_{N}}$ is reduced for any $c$-admissible sequence $x_{1} \cdots x_{N}$. Consider the sequence $u_{k}=\left(\pi_{1} \pi_{2} \cdots \pi_{n}\right)^{k} e$. Clearly, the sequence $\ell\left(u_{1}\right)$, $\ell\left(u_{2}\right), \ldots$ is weakly increasing. We claim that in fact it is strictly increasing. If not, there is some $u=u_{k}=u_{k+1}$ with $\pi_{1} u=\pi_{2} u=\cdots=\pi_{n} u=u$. But then $s_{i}$ is an inversion of $u$ for every $i$ from 1 to $n$. In an infinite Coxeter group, there is no 
element with this property. (In a finite Coxeter group, the only element with this property is the maximal element $w_{0}$.)

Therefore, $\ell\left(u_{k}\right) \geq k$. By Proposition 3.1 there is a $c$-admissible reduced word for each $u_{k}$; call this reduced word $\mathrm{w}_{k}$. We know that $\mathrm{w}_{k}$ has length at least $k$. Let $z$ be the letter that occurs most often in $\mathrm{w}_{k}$; then $\phi(\mathrm{w})_{z} \geq k / n$. (Recall the map $\phi$ from Section 2,) Now we use that $\Gamma$ is connected. Let $\delta$ be the diameter of the graph $\Gamma$. If $x$ and $y$ are adjacent vertices of $\Gamma$, then $x$ and $y$ alternate within $\mathrm{w}_{k}$, so $\left|\phi\left(\mathrm{w}_{k}\right)_{x}-\phi\left(\mathrm{w}_{k}\right)_{y}\right| \leq 1$ and we deduce that $\phi\left(\mathrm{w}_{k}\right)_{x} \geq k / n-\delta$ for any $x$. Let $M$ be the greatest number of times any letter occurs in $x_{1} \cdots x_{N}$. Choosing $k$ large enough that $k / n-\delta \geq M$, we see that $\phi\left(\mathrm{w}_{k}\right)_{x} \geq \phi\left(x_{1} \cdots x_{N}\right)_{x}$ for any $x$. So, by Proposition [2.2, $x_{1} \cdots x_{N}$ is equivalent to a prefix of the reduced word $\mathrm{w}_{k}$. In particular, $s_{x_{1}} \cdots s_{x_{N}}$ is reduced. This concludes the proof of Theorem 2 and hence proves Theorem 1 .

We note one variant of this argument.

Theorem 3. Let $W$ be a finite Coxeter group. Then there is a c-admissible sequence $x_{1} x_{2} \cdots x_{N}$ such that $s_{x_{1}} s_{x_{2}} \cdots s_{x_{N}}$ is a reduced word for $w_{0}$.

Proof. Define $u_{k}=\left(\pi_{1} \pi_{2} \cdots \pi_{n}\right)^{k} e$ as before. We must have $u_{k}=w_{0}$ for $k$ sufficiently large. So there is a $c$-admissible sequence with Demazure product $w_{0}$. By Proposition 3.1. the shortest such $c$-admissible sequence gives a reduced word for $w_{0}$.

In the introduction, because we lacked the terminology of admissible sequences, we stated this result differently. The following corollary makes the connection.

Corollary 4.1. It is possible to interchange labels of commuting reflections in the semi-infinite sequence $c^{\infty}:=12 \cdots n 12 \cdots n \cdots$ so that the $c$-admissible sequence $x_{1} x_{2} \cdots x_{N}$ from Theorem 3 becomes a prefix of the resulting semi-infinite word.

Proof. Let $M$ be a positive integer which is larger than the number of times any given letter occurs in the word $x_{1} x_{2} \cdots x_{N}$. Then, by Proposition 2.2, the word $x_{1} x_{2} \cdots x_{N}$ is equivalent to a prefix of $(12 \cdots n)^{M}$. Adding additional letters to the right of $(12 \cdots n)^{M}$ does not change this.

Note that we can start with the semi-infinite word $A$ of which $x_{1} x_{2} \cdots x_{N}$ is a prefix and undo all of the commutations of commuting generators, keeping track of where the first $N$ letters go. We thus obtain a subword of $c^{\infty}$ which gives a reduced word for $w_{0}$. Consider any generator $s_{i} \in S$. There is some integer $k$ such that the first $k$ occurrences of $i$ in w are in the prefix $x_{1} x_{2} \cdots x_{N}$, after which none of the later occurrences of $i$ in $A$ lie in $x_{1} x_{2} \cdots x_{N}$. Without loss of generality, we may assume that we never interchange $i$ with itself while transforming $A$ into $c^{\infty}$. Thus, we have obtained a subword $\mathbf{w}_{\mathbf{0}}$ of $c^{\infty}$ such that $\mathbf{w}_{\mathbf{0}}$ is a reduced word for $w_{0}$ and, for each $s_{i} \in S$, there is some $k$ such that the first $k$ occurrences of $i$ in $c^{\infty}$ lie in $\mathbf{w}_{\mathbf{0}}$, while all the later occurrences of $i$ do not. In other words, $w_{0}$ is $c$-sortable in the sense of [10. This gives an efficient proof of Corollary 4.4 of [10] without any case by case analysis. The reduced word $\mathbf{w}_{\mathbf{0}}$ plays an important role in [3]. Theorem 3 , and its proof, may be of use in finding better descriptions of $\mathbf{w}_{\mathbf{0}}$.

\section{Acknowledgments}

I am grateful to Nathan Reading for making me aware of the work of Kleiner and Pelley, as well as several other references cited here. Christophe Hohlweg, Carsten 
Lange and Hugh Thomas showed me preliminary drafts of $[3]$ and were very helpful in responding to my questions about it. Andrei Zelevinsky and Mark Kleiner both encouraged me to pursue a purely combinatorial proof. Daan Krammer helped guide me through the results of 8 . The anonymous referee corrected an error in the original statement of Proposition 1.1 and made many other helpful suggestions. I was funded during this research by a research fellowship from the Clay Mathematics Institute.

\section{REFERENCES}

[1] A. Björner and F. Brenti, Combinatorics of Coxeter Groups, Graduate Texts in Mathematics 231, Springer-Verlag, 2005. MR2133266 (2006d:05001)

[2] S. Fomin and A. Zelevinsky, Cluster algebras IV, Coefficients, Compositio Mathematica 143 (2007), 112-164. MR2295199 (2008d:16049)

[3] C. Hohlweg, C. Lange and H. Thomas, Permutahedra and Generalized Associahedra, arXiv:0709.4241

[4] R. B. Howlett, Coxeter groups and $M$-matrices, Bulletin of the London Mathematical Society 14 (1982), no. 2, 137-141. MR647197 (83g:20032)

[5] M. Kleiner and A. Pelley, Admissible sequences, preprojective representations of quivers, and reduced words in the Weyl group of a Kac-Moody algebra, International Mathematics Research Notices (2007), no. 4, Art. ID mm013. MR2338197 (2008f:16035)

[6] M. Kleiner and H. R. Tyler, Admissible sequences and the preprojective component of a quiver, Advances in Mathematics 192 (2005), no. 2, 376-402. MR2128704 (2006d:16026)

[7] A. Knutson and E. Miller, Subword complexes in Coxeter groups, Advances in Mathematics 184 (2004), no. 1, 161-176. MR2047852(2005c:20066)

[8] D. Krammer, The conjugacy problem for Coxeter groups, Ph.D. thesis, Universiteit Utrecht, 1994. Available at http://www.warwick.ac.uk/ masbal/

[9] A. Kuniba, K. Misra, M. Okado, T. Takagi, and J. Uchiyama, Crystals for Demazure modules of classical affine Lie algebras, Journal of Algebra 208 (1998), no. 1, 185-215. MR1643999 (99h:17008)

[10] N. Reading, Cambrian Lattices, Advances in Mathematics 205 (2006), no. 2, 313-353. MR2258260 (2007g:05195)

[11] N. Reading, Sortable elements and Cambrian lattices, Algebra Universalis 56 (2007), no. 3-4, 411-437. MR2318219(2008d:20073)

[12] N. Reading and D. Speyer, Cambrian Fans, JEMS to appear, arXiv:math.C0/0606201.

[13] N. Reading and D. Speyer, Sortable elements in infinite Coxeter groups, arXiv:0803.2722.

Department of Mathematics, Massachusetts Institute of Technology, 77 MassachuSetts Avenue, Cambridge, Massachusetts 02139

E-mail address: speyer@math.mit.edu 